\title{
Robust Filtering with Stochastic Nonlinearities and Multiple Missing Measurements *
}

\author{
Guoliang Wei ${ }^{\text {a }}$, Zidong Wang ${ }^{\text {a,b }}$, Huisheng Shu ${ }^{\text {a }}$ \\ ${ }^{\text {a } S c h o o l ~ o f ~ I n f o r m a t i o n ~ S c i e n c e ~ a n d ~ T e c h n o l o g y, ~ D o n g h u a ~ U n i v e r s i t y, ~ S h a n g h a i ~ 200051, ~ C h i n a . ~}$ \\ ${ }^{\mathrm{b}}$ Department of Information Systems and Computing, Brunel University, Uxbridge, Middlesex, UB8 3PH, U.K.
}

\begin{abstract}
This paper is concerned with the filtering problem for a class of discrete-time uncertain stochastic nonlinear time-delay systems with both the probabilistic missing measurements and external stochastic disturbances. The measurement missing phenomenon is assumed to occur in a random way, and the missing probability for each sensor is governed by an individual random variable satisfying a certain probabilistic distribution over the interval [0 1]. Such a probabilistic distribution could be

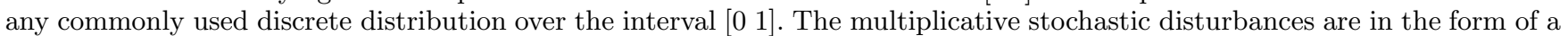
scalar Gaussian white noise with unit variance. The purpose of the addressed filtering problem is to design a filter such that, for the admissible random measurement missing, stochastic disturbances, norm-bounded uncertainties as well as stochastic nonlinearities, the error dynamics of the filtering process is exponentially mean-square stable. By using the linear matrix inequality (LMI) method, sufficient conditions are established that ensure the exponential mean-square stability of the filtering error, and then the filter parameters are characterized by the solution to a set of LMIs. Illustrative examples are exploited to show the effectiveness of the proposed design procedures.
\end{abstract}

Key words: Stochastic systems; nonlinear systems; uncertain systems; time-delay; missing measurements.

\section{Introduction}

In the past few years, stochastic modeling has come to play an important role in many branches of science such as biology, economics and engineering applications. Linear systems with stochastic perturbations have been intensively investigated from researchers working in different areas, see $[2,3]$ and references therein. Since nonlinearities are inevitable in most real-world systems, analysis and synthesis of nonlinear stochastic systems have been attracting more and more research attention, see e.g. $[9,16]$. Note that, the kind of stochastic nonlinearities described by statistical means has drawn particular research focus since it covers several well-studied nonlinearities in stochastic systems, see [18]. On the other hand, the robust filtering and control problems for stochastic systems with both time delays and parameter uncertainties have also received considerable research attention, see e.g. $[5,9,10,12]$ for some sample publications.

* This work was supported in part by the Shanghai Natural Science Foundation under Grant 07ZR14002, the Engineering and Physical Sciences Research Council (EPSRC) of the U.K. under Grant GR/S27658/01, an International Joint Project sponsored by the Royal Society of the U.K., the Nuffield Foundation of the U.K. under Grant NAL/00630/G and the Alexander von Humboldt Foundation of Germany. Corresponding author Zidong Wang.

Email address: Zidong.Wang@brunel.ac.uk (Zidong Wang).
The missing measurement phenomenon usually occurs in networked control systems and has attracted considerable attention during the past few years, see e.g. [4,13,14] and the references therein. The binary random variable sequence (also called Bernoulli distributed model) has been a popular approach to modeling the missing observation phenomenon. Such a model was employed as early as in [11] to synthesize the recursive filters for systems with missing measurement. Recently, with the rapid development of networked control systems, renewed efforts have been devoted to this effective and flexible model, see e.g. $[14,15]$.

It should be pointed out that, in all the aforementioned literature, the probability 0 is used to stand for an entire signal missing and the probability 1 denotes the intactness (i.e., there is no signal missing at all), and all the sensors have the same missing probability. Such a description, however, does have its limitations since it cannot cover some practical cases, for example, the case when only partial information is missing and the case when the individual sensor has different missing probability. Note that the latter case has been dealt with in $[6,7]$ where the minimum variance state estimators have been designed for linear systems with multiple sensors with different failure/delay rates. However, to the best of the authors' knowledge, the filtering problem has not yet been addressed for uncertain stochastic nonlinear time-delay systems with probabilistic missing measurements, which still remains as a challenging problem. 
In this paper, the filtering problems are addressed for a class of discrete-time stochastic nonlinear time-delay systems with sensors information dropout and stochastic disturbances. The sensor measurement missing is assumed to be random and different for individual sensor, which is modeled by individual random variable satisfying a certain probabilistic distribution on the interval [0 1]. Such a probabilistic distribution could be any commonly used discrete distributions. The multiplicative stochastic disturbances are in the form of a scalar Gaussian white noise with unit variance. We are interested in designing a filter such that the overall filtering error is exponentially mean-square stable. By using the linear matrix inequality (LMI) method, sufficient conditions are derived to ensure the existence of the desired filters which are then characterized by the solution to a set of LMIs. Illustrative examples are exploited to show the effectiveness of the proposed design procedures.

Notations: In this paper, $\mathbb{R}^{n}, \mathbb{R}^{n \times m}$ and $\mathbb{Z}^{+}$denote, respectively, the $n$-dimensional Euclidean space, the set of all $n \times m$ real matrices and the set of nonnegative integers. $I$ denote the identity matrix of compatible dimension. The notation $X \geq Y$ (respectively, $X>Y$ ), where $X$ and $Y$ are symmetric matrices, means that $X-Y$ is positive semi-definite (respectively, positive definite). $M^{T}$ represents the transpose of $M$. When $x$ is a stochastic variable, $\mathbb{E}\{x\}$ stands for the expectation of $x$. The shorthand $\operatorname{diag}\left\{M_{1}, M_{2}, \ldots, M_{n}\right\}$ denotes a block diagonal matrix with diagonal blocks being the matrices $M_{1}, M_{2}, \ldots, M_{n}$ and is denoted as $\operatorname{diag}_{n}\{M\}$ when $M_{1}=M_{2}=\cdots=M_{n}$. In symmetric block matrices, the symbol $*$ is used as an ellipsis for terms induced by symmetry. Matrices, if they are not explicitly stated, are assumed to have compatible dimensions.

\section{Problem Formulation}

Consider the following discrete-time uncertain stochastic system with state-delay and stochastic nonlinearities:

$$
\left\{\begin{aligned}
x(k+1)= & A(k) x(k)+A_{d}(k) x_{d}(k)+f(k, d) \\
& +E_{1} x(k) \omega(k) \\
x(k)= & \rho(k), \quad k=-d,-d+1, \ldots, 0
\end{aligned}\right.
$$

where $x(k) \in \mathbb{R}^{n}$ is the state; $d \in \mathbb{Z}^{+}$is a known constant time-delay with $d \geq 1 . \omega(k)$ is an one-dimensional Gaussian white noise sequence satisfying $\mathbb{E}\{\omega(k)\}=0$ and $\mathbb{E}\left\{\omega^{2}(k)\right\}=1 . \rho(k)$ is the initial state of the system. $A(k)=A+\Delta A(k), A_{d}(k)=A_{d}+\Delta A_{d}(k)$ with $\Delta A(k)$ and $\Delta A_{d}(k)$ are unknown matrices representing parameter uncertainties in the following form

$$
\left[\Delta A(k) \Delta A_{d}(k)\right]=M F(k)\left[\begin{array}{ll}
N & N_{d}
\end{array}\right]
$$

with $F(k)$ satisfying $F^{T}(k) F(k) \leq I$.

The measurement with sensor data missing is described by

$$
\begin{aligned}
y(k) & =\Xi C x(k)+g(k, d)+E_{2} x(k) \omega(k) \\
& =\sum_{i=1}^{m} \xi_{i} C_{i} x(k)+g(k, d)+E_{2} x(k) \omega(k),
\end{aligned}
$$

where $y(k) \in \mathbb{R}^{m}$ is the measured output vector, $\Xi=$ $\operatorname{diag}\left\{\xi_{1}, \cdots, \xi_{m}\right\}$ with $\xi_{i}(i=1, \ldots, m)$ being $m$ unrelated random variables which are also unrelated with $\omega(k)$. It is assumed that $\xi_{i}$ has the probabilistic density function $p_{i}(s)(i=1, \ldots, m)$ on the interval [0 1] with mathematical expectation $\mu_{i}$ and variance $\sigma_{i}^{2} . C_{i}:=$ $\operatorname{diag}\{\underbrace{0, \cdots, 0}_{i-1}, 1, \underbrace{0, \cdots, 0}_{m-i}\} C(i=1, \ldots, m)$, and $A, A_{d}$, $C, E_{1}, E_{2}, M, N$ and $N_{d}$ are known constant matrices.

Remark 1 Equation (3) describes the measurement with multiple sensors, in which the diagonal matrix $\Xi$ represents the whole missing statues and the random variable $\xi_{i}$ corresponds to the ith sensor $(i=1, \ldots, m)$. Note that the measurement missing phenomenon has been extensively considered and several models have been introduced. One popular model is arguably the Bernoulli distributed model in which 0 is used to stand for an entire missing of signals and 1 denotes the intactness, see e.g. $[14,15]$. However, in practice, for a variety of reasons such as sensor aging or sensor partial failure, the information transmitted at one moment from/to a sensor could be neither completely missing nor completely successful, but only part of the information can go through. Such a case has been extensively studied in the literature, see [8,17] and the reference therein. In the networked control terminology, this could also be referred to as the information quantization. In other words, the proportion of the data missed at one moment could be a fraction other than 0 or 1 . In (3), $\xi_{i}$ can take value on the interval [ $\left[\begin{array}{ll}0 & 1\end{array}\right]$ and the probability for $\xi_{i}$ to take different values may be different. $\xi_{i}$ can satisfy any discrete probabilistic distributions on the interval [0 1], and therefore includes the Bernoulli distribution as a special case.

The functions $f(k, d)$ and $g(k, d)$ describe the so-called stochastic nonlinear functions of the states and delayed states, which are bounded in a statistical sense as follows:

$$
\begin{aligned}
& \mathbb{E}\left\{\left[\begin{array}{l}
f(k, d) \\
g(k, d)
\end{array}\right] \mid x(k)\right\}=0, \\
& \mathbb{E}\left\{\left[\begin{array}{l}
f(k, d) \\
g(k, d)
\end{array}\right]\left[f^{T}(l, d) g^{T}(l, d)\right] \mid x(l)\right\}=0, k \neq l, \quad(5) \\
& \mathbb{E}\left\{\left[\begin{array}{l}
f(k, d) \\
g(k, d)
\end{array}\right]\left[f^{T}(k, d) g^{T}(k, d)\right] \mid x(k)\right\} \leq \sum_{i=1}^{q}\left[\begin{array}{l}
\pi_{1 i} \\
\pi_{2 i}
\end{array}\right] \\
& \quad \times\left[\begin{array}{l}
\pi_{1 i} \\
\pi_{2 i}
\end{array}\right]^{T}\left[x^{T}(k) \Phi_{i} x(k)+x_{d}^{T}(k) \Psi_{i} x_{d}(k)\right]
\end{aligned}
$$

where $q$ is a known nonnegative integer. $\pi_{1 i} \in \mathbb{R}^{n \times 1}$ and $\pi_{2 i} \in \mathbb{R}^{m \times 1}(i=1, \ldots, q)$ have compatible dimensions with $f(k, d)$ and $g(k, d)$, and $\Phi_{i}$ and $\Psi_{i}(i=1, \ldots, q)$ are positive-definite matrices with appropriate dimensions. For convenience, we assume that $f(k, d)$ and $g(k, d)$ are unrelated with $\xi_{i}(i=1, \ldots, m)$ and $\omega(k)$.

Remark 2 The so-called stochastic nonlinearities described in (4)-(6) have been extensively considered (see [18] and the references therein) since such a description covers several well-studied nonlinear functions as special 
cases. It should be noted that, in this paper, such nonlinear functions involve delayed states and are therefore more general that those in [18].

In this paper, we are interested in designing a linear filter of the following structure:

$x_{f}(k+1)=A_{f} x_{f}(k)+K y(k)$,

where $x_{f}(k) \in \mathbb{R}^{n}$ is the state estimate, and $A_{f}$ and $K$ are filter parameters to be determined.

By augmenting the state variables

$\tilde{x}(k)=\left[\begin{array}{c}x(k) \\ x_{f}(k)\end{array}\right], \tilde{x}_{d}(k)=\left[\begin{array}{c}x_{d}(k) \\ x_{f d}(k)\end{array}\right], h(k)=\left[\begin{array}{c}f(k, d) \\ g(k, d)\end{array}\right]$

and combining (1) and (7), we obtain the filtering error dynamics as follows:

$$
\begin{aligned}
\tilde{x}(k+1)= & \mathcal{A} \tilde{x}(k)+\mathcal{A}_{d} Z \tilde{x}_{d}(k)+\mathcal{C} \tilde{x}(k)+\mathcal{B} h(k) \\
& +\mathcal{E} Z \tilde{x}(k) \omega(k),
\end{aligned}
$$

where

$$
\begin{aligned}
\mathcal{A} & =\left[\begin{array}{cc}
A+\Delta A(k) & 0 \\
K \bar{\Xi} C & A_{f}
\end{array}\right], \mathcal{C}=\left[\begin{array}{cr}
0 & 0 \\
K(\Xi-\bar{\Xi}) C & 0
\end{array}\right], \\
\mathcal{A}_{d} & =\left[\begin{array}{c}
A_{d}+\Delta A_{d}(k) \\
0
\end{array}\right], \mathcal{B}=\left[\begin{array}{ll}
I & 0 \\
0 & K
\end{array}\right], \mathcal{E}=\left[\begin{array}{c}
E_{1} \\
K E_{2}
\end{array}\right], \\
Z & =\left[\begin{array}{ll}
I & 0
\end{array}\right], \bar{\Xi}=\mathbb{E}\{\Xi\} .
\end{aligned}
$$

Observe the system $(8)$ and let $\tilde{x}(k ; \nu)$ denote the state trajectory from the initial data $\tilde{x}(s)=\nu(s)$ on $-d \leq s \leq$ 0 . Obviously, $\tilde{x}(k, 0) \equiv 0$ is the trivial solution of system (8) corresponding to the initial data $\nu=0$.

In this paper, we aim to design an linear filter of the form (7) for the system (1) such that, for all admissible time delay, uncertainties, sensors data missing, stochastic nonlinearities and exogenous stochastic disturbances, the filtering error system (8) is exponentially mean-square stable.

\section{Main Results}

\subsection{Filter analysis}

Lemma 1 (S-procedure) [1] Let $\Upsilon=\Upsilon^{T}, \mathcal{M}$ and $\mathcal{N}$ be real matrices of appropriate dimensions with $F$ satisfying $F^{T} F \leq I$, then

$$
\Upsilon+\mathcal{M F \mathcal { N }}+\mathcal{N}^{T} F^{T} \mathcal{M}^{T}<0
$$

if and only if there exists a positive scalar $\delta>0$ such that

$$
\Upsilon+\frac{1}{\delta} \mathcal{M} \mathcal{M}^{T}+\delta \mathcal{N}^{T} \mathcal{N}<0,
$$

or, equivalently

$$
\left[\begin{array}{ccc}
\Upsilon & \mathcal{M} & \delta \mathcal{N}^{T} \\
\mathcal{M}^{T} & -\delta I & 0 \\
\delta \mathcal{N} & 0 & -\delta I
\end{array}\right]<0
$$

For convenience of presentation, we firstly deal with the nominal system of (1) (i.e. without parameter uncertainties) and will eventually extend our main results to include the robustness. In the following theorem, Lyapunov stability theorem and an LMI-based method are combined together to deal with the stability analysis problem for the filter design of the discrete-time stochastic nonlinear system with time delay and stochastic disturbance. A sufficient condition is derived that guarantees the solvability of the exponential filtering problem.

Theorem 1 Consider the augmented filtering system (8) with given filter parameters. If there exist positive definite matrices $P>0, Q>0$ and positive scalars $\varepsilon_{i}>0(i=1, \ldots, q)$ such that the following matrix inequalities

$$
\left[\begin{array}{ccccccc}
-P+Z^{T} Q Z & * & * & * & * & * & * \\
0 & -Q & * & * & * & * & * \\
P \mathcal{A} & P \mathcal{A}_{d} & -P & * & * & * & * \\
\overline{\mathcal{C}} & 0 & 0 & -\mathcal{P} & * & * & * \\
P \mathcal{E} Z & 0 & 0 & 0 & -P & * & * \\
\hat{\Phi} & 0 & 0 & 0 & 0 & -\Lambda & * \\
0 & \hat{\Psi} & 0 & 0 & 0 & 0 & -\Lambda
\end{array}\right]<0,
$$

hold, where

$$
\begin{aligned}
\overline{\mathcal{C}} & :=\left[\sigma_{1} \overline{\mathcal{C}}_{1}^{T} P, \cdots, \sigma_{m} \overline{\mathcal{C}}_{m}^{T} P\right]^{T}, \mathcal{P}:=\operatorname{diag}\{P, \cdots, P\} \\
\hat{\Phi} & :=\left[\varepsilon_{1} \bar{\Phi}_{1}^{\frac{1}{2}}, \cdots, \varepsilon_{q} \bar{\Phi}_{q}^{\frac{1}{2}}\right]^{T}, \hat{\Psi}:=\left[\varepsilon_{1} \Psi_{1}^{\frac{1}{2}}, \cdots, \varepsilon_{q} \Psi_{q}^{\frac{1}{2}}\right]^{T},(11 \\
\bar{\Phi}_{i} & :=\left[\begin{array}{cc}
\Phi_{i} & 0 \\
0 & 0
\end{array}\right], \bar{\Pi}_{i}:=\left[\begin{array}{c}
\pi_{1 i} \\
\pi_{2 i}
\end{array}\right], \overline{\mathcal{C}}_{i}=\left[\begin{array}{cc}
0 & 0 \\
K C_{i} & 0
\end{array}\right], \\
\Lambda & :=\operatorname{diag}\left\{\varepsilon_{1} I, \cdots, \varepsilon_{q} I\right\},
\end{aligned}
$$

then the filtering error system (8) is exponentially meansquare stable.

Proof: Define the following Lyapunov functional candidate for the system (8):

$$
V(\tilde{x}(k), k)=\tilde{x}^{T}(k) P \tilde{x}(k)+\sum_{s=k-d}^{k-1} \tilde{x}^{T}(s) Z^{T} Q Z \tilde{x}(s) .(1
$$

From the definition of $\mathcal{C}$, we can easily know $\mathbb{E} \mathcal{C}=0$. Furthermore, from the fact $\mathbb{E} \omega(k)=0$ and (4)-(5), calculating the difference of the Lyapunov functional (12) according to $(8)$ gives

$$
\begin{aligned}
& \mathbb{E}\{\Delta V(\tilde{x}(k), k)\} \\
= & {\left[\mathcal{A} \tilde{x}(k)+\mathcal{A}_{d} Z \tilde{x}_{d}(k)\right]^{T} P\left[\mathcal{A} \tilde{x}(k)+\mathcal{A}_{d} Z \tilde{x}_{d}(k)\right] } \\
& +\mathbb{E}\left\{\omega^{T}(k) \tilde{x}^{T}(k) Z \mathcal{E}^{T} P \mathcal{E} Z \tilde{x}(k) \omega(k)\right\}-\tilde{x}^{T}(k) P \tilde{x}(k) \\
& +\mathbb{E}\left\{\tilde{x}^{T}(k) \mathcal{C}^{T} P \mathcal{C} \tilde{x}(k)\right\}+\mathbb{E}\left\{h^{T}(k) \mathcal{B}^{T} P \mathcal{B} h(k)\right\} \\
& +\tilde{x}^{T}(k) Z^{T} Q Z \tilde{x}(k)-\tilde{x}_{d}^{T}(k) Z^{T} Q Z \tilde{x}_{d}(k),
\end{aligned}
$$


We can obtain from the definition of (3) that

$\mathbb{E}\left\{\mathcal{C}^{T} P \mathcal{C}\right\}=\sum_{i=1}^{m} \sigma_{i}^{2} \overline{\mathcal{C}}_{i}^{T} P \overline{\mathcal{C}}_{i}$

Again, we can have from (4)-(6) that

$$
\begin{array}{r}
\mathbb{E}\left\{h^{T}(k) \mathcal{B}^{T} P \mathcal{B} h(k)\right\} \leq \sum_{i=1}^{q}\left[\tilde{x}^{T}(k) \bar{\Phi}_{i} \tilde{x}(k)\right. \\
\left.+\tilde{x}_{d}^{T}(k) Z^{T} \Psi_{i} Z \tilde{x}_{d}(k)\right] \operatorname{tr}\left(\mathcal{B} \Pi_{i} \mathcal{B}^{T} P\right),
\end{array}
$$

where $\Pi_{i}:=\bar{\Pi}_{i} \bar{\Pi}_{i}^{T}$ with $\bar{\Pi}_{i}$ and $\bar{\Phi}_{i}(i=1, \ldots, q)$ being defined in (11).

From (13)-(15), we have

$\mathbb{E}\{\Delta V(\tilde{x}(k), k)\} \leq \mathbb{E}\left\{\eta^{T}(k) \Omega \eta(k)\right\}$,

where $\eta(k)=\left[\begin{array}{lll}\tilde{x}^{T}(k) & Z \tilde{x}_{d}^{T}(k)\end{array}\right]$ and

$\Omega:=\left[\begin{array}{cc}\Omega_{1} & \mathcal{A}^{T} P \mathcal{A}_{d} \\ \mathcal{A}_{d}^{T} P \mathcal{A} & -\Omega_{2}\end{array}\right]$,

with $\Omega_{1}:=-P+\sum_{i=1}^{m} \sigma_{i}^{2} \overline{\mathcal{C}}_{i}^{T} P \overline{\mathcal{C}}_{i}+Z^{T} \mathcal{E}^{T} P \mathcal{E} Z+Z^{T} Q Z+$ $\mathcal{A}^{T} P \mathcal{A}+\sum_{i=1}^{q} \bar{\Phi}_{i} \operatorname{tr}\left(\mathcal{B} \Pi_{i} \mathcal{B}^{T} P\right), \Omega_{2}:=Q-\mathcal{A}_{d}^{T} P \mathcal{A}_{d}-$ $\sum_{i=1}^{q} \Psi_{i} \operatorname{tr}\left(\mathcal{B} \Pi_{i} \mathcal{B}^{T} P\right)$.

By Schur complement, (10) holds if and only if $\operatorname{tr}\left(\mathcal{B} \Pi_{i} \mathcal{B}^{T} P\right)$

$<\varepsilon_{i}(i=1, \ldots, q)$. By Schur complement, we can obtain from (9) and (10) that $\Omega<0$ and, subsequently,

$\mathbb{E}\{\Delta V(\tilde{x}(k))\}<-\lambda_{\min }(\Omega)|\tilde{x}(k)|^{2}$.

where $\lambda_{\min }(\Omega)$ is the minimum eigenvalue of $\Omega$. Finally, we can confirm from Lemma 1 of [14] that the augmented filtering systems (8) is exponentially mean-square stable.

In the next subsection, our attention is focused on the design of filter parameters $A_{f}$ and $K$ by using the results in Theorem 1.

\subsection{Filter synthesis}

Theorem 2 Consider the system (8). If there exist positive-definite matrices $S>0, R>0, Q>0$, matrices $\tilde{K}, \tilde{A}_{f}$ and positive scalars $\varepsilon_{i}>0,(i=1,2, \ldots, q)$ such that the following linear matrix inequalities

$$
\begin{gathered}
\Upsilon:=\left[\begin{array}{ccccccc}
-\Upsilon_{11} & * & * & * & * & * & * \\
0 & -Q & * & * & * & * & * \\
\Upsilon_{31} & S A_{d} & -\$ & * & * & * & * \\
\Upsilon_{41} & 0 & 0 & -\overline{\mathcal{P}} & * & * & * \\
\Upsilon_{51} & 0 & 0 & 0 & -\$ & * & * \\
\Upsilon_{61} & 0 & 0 & 0 & 0 & -\Lambda & * \\
0 & \hat{\Psi} & 0 & 0 & 0 & 0 & -\Lambda
\end{array}\right]<0, \\
{\left[\begin{array}{ccc}
-\varepsilon_{i} & * & * \\
S \pi_{1 i} & -S & * \\
R \pi_{1 i}+\tilde{K} \pi_{2 i} & -S & -R
\end{array}\right]<0, i=1, \ldots, q,} \\
{\left[\begin{array}{c}
S-R>0,
\end{array}\right.}
\end{gathered}
$$

hold, where

$$
\begin{aligned}
& \tilde{\mathcal{C}}:=\left[\left[\begin{array}{c}
0 \\
\sigma_{1} \tilde{K} C_{1}
\end{array}\right]^{T}, \cdots,\left[\begin{array}{c}
0 \\
\sigma_{m} \tilde{K} C_{m}
\end{array}\right]^{T}\right]^{T}, \\
& \tilde{\Phi}:=\left[\left[\begin{array}{c}
\varepsilon_{1} \Phi_{1}^{\frac{1}{2}} \\
0
\end{array}\right]^{T}, \cdots,\left[\begin{array}{c}
\varepsilon_{q} \Phi_{q}^{\frac{1}{2}} \\
0
\end{array}\right]\right]^{T}, \\
& \$:=\left[\begin{array}{ll}
S & S \\
S & R
\end{array}\right], \Upsilon_{11}:=\$-\left[\begin{array}{ll}
Q & Q \\
Q & Q
\end{array}\right], \\
& \Upsilon_{31}:=\left[\begin{array}{cc}
S A & S A \\
R A+\tilde{K} \bar{\Xi} C+\tilde{A}_{f} & R A+\tilde{K} \bar{\Xi} C
\end{array}\right] \text {, } \\
& \Upsilon_{41}:=\left[\begin{array}{ll}
\tilde{\mathcal{C}} & \tilde{\mathcal{C}}
\end{array}\right], \overline{\mathcal{P}}:=\operatorname{diag}_{m}\{\$\}, \Upsilon_{61}:=\left[\begin{array}{ll}
\tilde{\Phi} & \tilde{\Phi}
\end{array}\right] \\
& \Upsilon_{51}:=\left[\begin{array}{cc}
S E_{1} & S E_{1} \\
R E_{1}+\tilde{K} E_{2} & R E_{1}+\tilde{K} E_{2}
\end{array}\right],
\end{aligned}
$$

then the system (8) is exponentially mean-square stable. In this case, the parameters of the desired filter (7) are given as follows:

$$
K:=X_{12}^{-1} \tilde{K}, A_{f}:=X_{12}^{-1} \tilde{A}_{f} S^{-1} Y_{12}^{-1},
$$

where $X_{12}, Y_{12}$ are any nonsingular matrices satisfying

$$
X_{12} Y_{12}^{T}=I-R S^{-1} \text {. }
$$

Proof: The proof is omitted owe to the limited space.

\subsection{The solution}

In the following theorem, we extend the main results in Theorem 2 to the parameter uncertain cases. The proof of this theorem can be obtained along the similar line of that of Theorem 2, and is therefore omitted here to avoid unnecessary duplication.

Theorem 3 Consider the system (8). If there exist positive-definite matrices $S>0, R>0, Q>0$, matrices $\tilde{K}, \tilde{A}_{f}$ and positive scalars $\delta>0$ and $\varepsilon_{i}>0$, $(i=1,2, \ldots, q)$ such that the linear matrix inequalities (20), (21) and

$$
\left[\begin{array}{ccc}
\Upsilon & * & * \\
\bar{\Upsilon}_{21} & -\delta I & * \\
\bar{\Upsilon}_{31} & 0 & -\delta I
\end{array}\right]<0,
$$

where $\bar{\Upsilon}_{21}:=\left[\begin{array}{llllllllll}0 & 0 & 0 & M^{T} S & M^{T} R & 0 & 0 & 0 & 0 & 0\end{array}\right], \bar{\Upsilon}_{31}:=$ $\left[\begin{array}{lllllllll}\delta N & \delta N & \delta N_{d} & 0 & 0 & 0 & 0 & 0 & 0\end{array}\right]$ hold, then the system (8) is exponentially mean-square stable.

Proof: The proof is also omitted because of the limited space.

Remark 3 In this paper, the discrete-time systems include the state-dependent external stochastic disturbances, stochastic nonlinearities (nonlinearities described by statistical means) as well as probabilistic sensor information missing. In practice, these three stochastic 'sources' make the real-time delay measurement a 
rather challenging task, which means the delay information is not easily accessible. Therefore, it makes sense to have delay-independent results. We point out that, in the case when the actually occurred delay is of small size and can be measured, it is technically not difficult to consider delay-dependent analysis by using recently developed techniques and reduce the possible conservatism.

\section{Numerical Example}

In this section, two simulation examples are presented to illustrate the usefulness and flexibility of the filter design method developed in this paper.

Example 1 In this example, we are interested in designing the filter for the discrete-time stochastic nonlinear uncertain system with stochastic disturbances and sensor data missing.

The system data of (1)-(3) are given as follows:

$$
\begin{gathered}
A=\left[\begin{array}{cc}
0.1 & 0 \\
0 & 0.2
\end{array}\right], \quad A_{d}=\left[\begin{array}{cc}
0.1 & 0 \\
0 & 0.1
\end{array}\right], C=\left[\begin{array}{ll}
1 & 0 \\
0 & 1
\end{array}\right], \\
E_{1}=\left[\begin{array}{cc}
0.4 & 0 \\
0 & 0.4
\end{array}\right], E_{2}=\left[\begin{array}{cc}
0.4 & 0 \\
0 & 0.1
\end{array}\right], M=\left[\begin{array}{l}
0.02 \\
0.02
\end{array}\right], \\
N=N_{d}=[0.010 .02], m=n=q=d=2 .
\end{gathered}
$$

The stochastic nonlinear functions are taken to satisfy

$$
\begin{aligned}
& \mathbb{E}\{\left.\left\{\begin{array}{l}
f(k, d) \\
g(k, d)
\end{array}\right] \mid x(k)\right\}=0, \\
& \mathbb{E}\left\{f(k, d) f^{T}(k, d) \mid x(k)\right\} \\
&= {\left[\begin{array}{l}
0.2 \\
0.2
\end{array}\right]\left[\begin{array}{l}
0.2 \\
0.2
\end{array}\right]^{T} x^{T}(k)\left[\begin{array}{cc}
0.11 & 0 \\
0 & 0.11
\end{array}\right] x(k) } \\
&+x_{d}^{T}(k)\left[\begin{array}{cc}
0.11 & 0 \\
0 & 0.11
\end{array}\right] x_{d}(k), \\
& \mathbb{E}\left\{g(k, d) g^{T}(k, d) \mid x(k)\right\} \\
&=\left[\begin{array}{c}
0.22 \\
0.22
\end{array}\right]\left[\begin{array}{c}
0.22 \\
0.22
\end{array}\right]^{T} x^{T}(k)\left[\begin{array}{cc}
0.11 & 0 \\
0 & 0.11
\end{array}\right] x(k) \\
& \quad+x_{d}^{T}(k)\left[\begin{array}{cc}
0.11 & 0 \\
0 & 0.11
\end{array}\right] x_{d}(k) .
\end{aligned}
$$

In addition, we assume the probabilistic density functions of $\xi_{1}$ and $\xi_{2}$ in $[0,1]$ described by

$$
p_{1}\left(s_{1}\right)=\left\{\begin{array}{ll}
0.8 & s_{1}=0 \\
0.1 & s_{1}=0.5 \\
0.1 & s_{1}=1
\end{array} \quad p_{2}\left(s_{2}\right)= \begin{cases}0.7 & s_{2}=0 \\
0.2 & s_{2}=0.5 \\
0.1 & s_{2}=1\end{cases}\right.
$$

from which the expectations and variances can be easily calculated as $\mu_{1}=0.15, \mu_{2}=0.2, \sigma_{1}^{2}=0.1025$ and $\sigma_{2}^{2}=0.11$.

Using Matlab LMI control Toolbox to solve the LMIs in (24), (20) and (21), we can calculate that the filter parameters as follows:
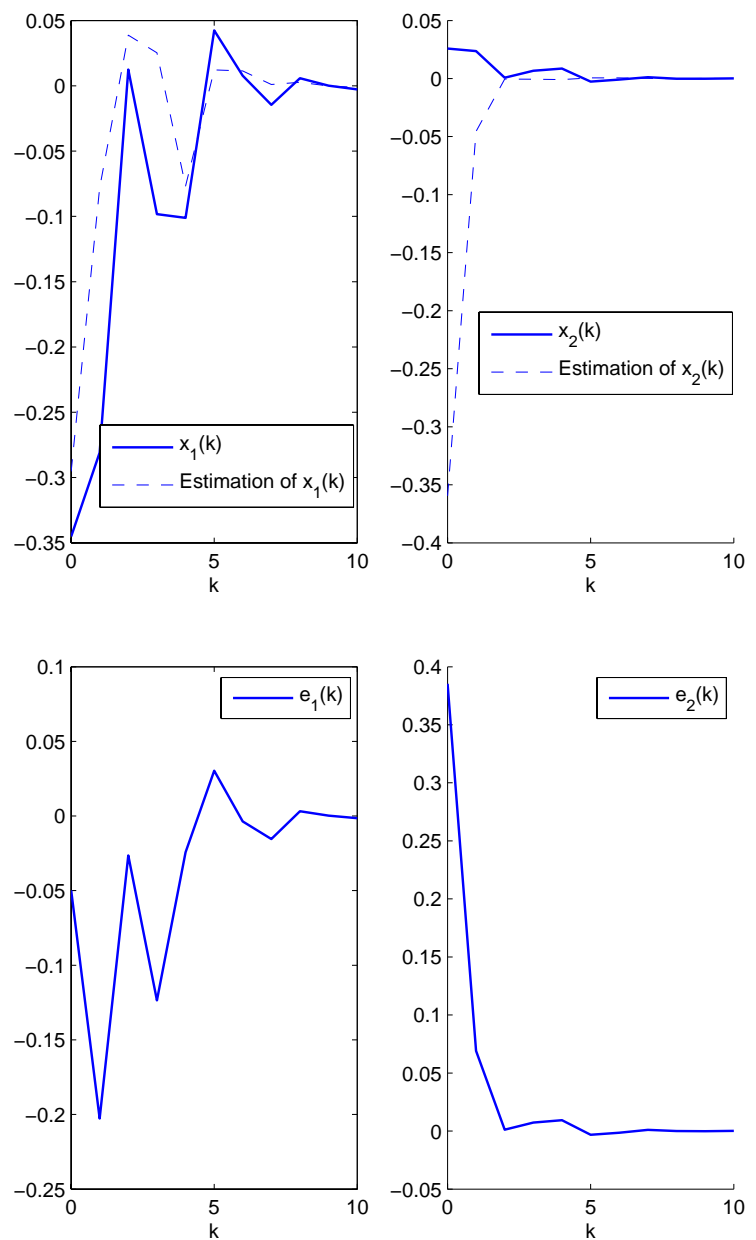

Fig. 1. The simulation results of Example 1

$$
K=\left[\begin{array}{ll}
0.4965 & 0.0249 \\
0.0189 & 0.3547
\end{array}\right], \quad A_{f}=\left[\begin{array}{cc}
0.0076 & -0.0070 \\
-0.0033 & 0.1198
\end{array}\right] \text {. }
$$

Fig. 1 displays the simulation results for the performance of the designed filter, in which e $(k)$ expresses the estimation error $e(k)=x(k)-x_{f}(k)$,

Example 2 In order to illustrate the effectiveness of our results for different measurement missing cases, for the same system as in Example 1, we assume that the probabilistic density functions of $\xi_{1}$ and $\xi_{2}$ in $[0,1]$ is given by

$$
p_{1}\left(s_{1}\right)=\left\{\begin{array}{ll}
0 & s_{1}=0 \\
0.1 & s_{1}=0.5 \\
0.9 & s_{1}=1
\end{array} p_{2}\left(s_{2}\right)= \begin{cases}0.1 & s_{2}=0 \\
0.1 & s_{2}=0.5 \\
0.8 & s_{2}=1\end{cases}\right.
$$

with the expectations and variances obtained as $\mu_{1}=$ 0.95, $\mu_{2}=0.85, \sigma_{1}^{2}=0.0225$ and $\sigma_{2}^{2}=0.1025$. Again, solving the LMIs in (24), (20) and (21) gives the filter parameters:

$$
K=\left[\begin{array}{ll}
0.5182 & 0.0316 \\
0.0289 & 0.3734
\end{array}\right], \quad A_{f}=\left[\begin{array}{ll}
-0.3115 & -0.0256 \\
-0.0229 & -0.0603
\end{array}\right] .
$$



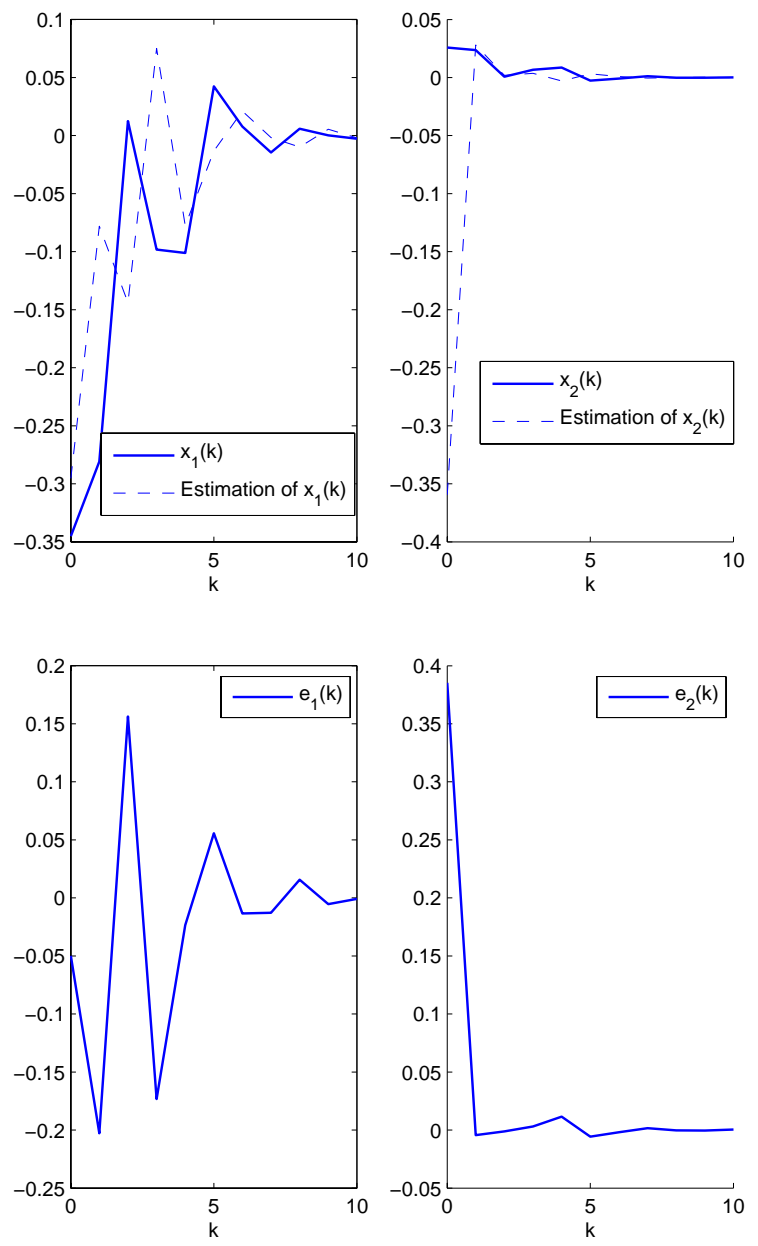

Fig. 2. The simulation results of Example 2

Fig. 2 shows the simulation results for the performance of the designed filter. We can see from the examples that different missing probability distributions give rise to different filters, all of which can accomplish the filtering task.

\section{Conclusions}

In this paper, the filtering problem has been studied for a class of discrete-time uncertain stochastic nonlinear time-delay systems with both the probabilistic missing measurements and external stochastic disturbances. The measurement missing phenomenon is assumed to occur in a random way, and the missing probability for each sensor is governed by an individual random variable satisfying a certain probabilistic distribution in the

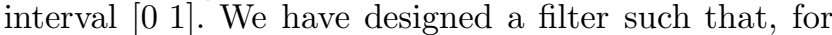
the admissible random measurement missing, stochastic disturbances, norm-bounded uncertainties as well as stochastic nonlinearities, the error dynamics of the filtering process is exponentially mean-square stable. Illustrative examples have been exploited to show the effectiveness of the proposed design procedures.

\section{References}

[1] S. Boyd, L. E. Ghaoui, E. Feron and V. Balakrishnan, Linear Matrix Inequalities in System and Control Theory, SIAM
Studies in Applied Mathematics, Philadelphia, 1994.

[2] H. Gao, J. Lam and C. Wang, Robust energy-to-peak filter design for stochastic time-delay systems, Systems $\&$ Control Letters, Vol. 55, No. 2, pp. 101-111, 2006.

[3] H. Gao, J. Lam, C. Wang and S. Xu, Robust $H_{\infty}$ filtering for 2D stochastic systems, Circuits, Systems and Signal Processing, Vol. 23, No. 6, pp. 479-505, 2004.

[4] H. Gao, and T. Chen, $H_{\infty}$ estimation for uncertain systems with limited communication capacity, IEEE Trans. Automatic Control. Vol. 52, No. 11, pp. 2070-2084, 2007.

[5] L. Guo, F. Yang and J. Fang, Multiobjective filtering for nonlinear time-delay systems with nonzero initial conditions based on convex optimizations, Circuits, Systems \&5 Signal Processing, Vol. 25, No. 5, pp. 591-607, 2006.

[6] F.O. Hounkpevi and E.E. Yaz, Robust minimum variance linear state estimators for multiple sensors with different failure rates, Auotmatica, Vol. 43, No. 7, pp. 1274-1280, 2007.

[7] F.O. Hounkpevi and E.E. Yaz, Minimum variance generalized state estimators for multiple sensors with different delay rates, Signal Processing, Vol. 87, No. 4, pp. 602-613, 2007.

[8] J. Liu, J.L. Wang and G.-H. Yang, Reliable guaranteed variance filtering against sensor failures, IEEE Trans. Signal Processing, Vol. 51, No. 5, pp. 1403-1411, 2003.

[9] Y. Liu, Z. Wang and X. Liu, Robust $H_{\infty}$ control for a class of nonlinear stochastic systems with mixed time-delay, Int. J. Robust \& Nonlinear Control, Vol. 17, No. 16, pp. 15251551, 2007.

[10] X. Lu, L. Xie, H. Zhang and W. Wang, Robust Kalman filtering for discrete-time systems with measurement delay, IEEE Trans. Circuits and Systems -II:Express Briefs, Vol. 54, No. 6, pp. 522-526, June. 2007.

[11] N. Nahi, Optimal recursive estimation with uncertain observation, IEEE Trans. Inform. Theory, Vol. 15, pp. 457462, Apr. 1969.

[12] Y. Niu, D.W.C. Ho and J. Lam, Robust integral sliding mode control for uncertain stochastic systems with time-varying delay, Automatica, Vol. 41, No. 5, pp. 873-880, 2005.

[13] Z. Wang, D. Ho and X. Liu, Variance-constrained filtering for uncertain stochastic systems with missing measurements, IEEE Trans. Automat. Control, Vol. 48, pp. 1254-1258, July 2003.

[14] Z. Wang and F. Yang, D. W. C. Ho and X. Liu, Robust $H_{\infty}$ filtering for stochastic time-delay systems with missing measurements, IEEE Trans. Signal Processing, Vol. 54, No. 7, pp. 2579-2587, 2006.

[15] Z. Wang, F. Yang, D. Ho and X. Liu, Robust $H_{\infty}$ control for networked systems with random packet losses, IEEE Trans. Systems, Man, and Cybernetics - Part B, Vol. 37, No. 4 pp. 916-924, 2007.

[16] Z. Wang, Y. Liu and X. Liu, $H_{\infty}$ filtering for uncertain stochastic time-delay systems with sector-bounded nonlinearities, Automatica, Vol. 44, No. 5, pp. 1268-1277, 2008.

[17] G.-H. Yang and D. Ye, Adaptive reliable $H_{\infty}$ filtering against sensor failures, IEEE Trans. Signal Processing, Vol. 55, No. 7, pp. 3161-3171, 2007.

[18] E. E. Yaz and Y. I. Yaz, State estimation of uncertain nonlinear stochastic systems with general criteria, Applied Mathematics Letters, Vol. 14, pp. 605-610, 2001. 